\title{
Microsatellite markers for regional differentiation of Puccinia graminis f. sp. tritici populations
}

\author{
Laprina Yu.V. ${ }^{*}$, Kelbin V.N. ${ }^{1}$, Skolotneva E.S. ${ }^{1}$, Kolomiets T.M. ${ }^{2}$, Kiseleva M.I. ${ }^{2}$, \\ Baranova O.A. ${ }^{3}$ \\ ${ }^{1}$ Institute of Cytology and Genetics, SB RAS, Novosibirsk, Russia \\ ${ }^{2}$ All-Russian Research Institute of Phytopathology, Moscow, Russia \\ ${ }^{3}$ All-Russian Institute of Plant Protection, St. Petersburg-Pushkin, Russia \\ *email: i.laprina@g.nsu.ru
}

The fungus Puccinia graminis f. sp. tritici (Pgt), which causes a devastating wheat disease, is airborne and spreads easily throughout the country. The high genetic plasticity of genome serves to increase the virulence of the stem rust population and overcome the resistant genes of the host plant. Genotyping of $P g t$ isolates can be a quick method to cover a larger sample. The aim of this study is to identify polymorphic microsatellite markers (SSRs, simple sequenced repeats) that differentiate regional Pgt populations. Sixteen SSR markers were screened against $P g t$ samples from non-black earth (NBE) and black earth (BE) areas of Russia. The amplicons were polymorphic, with 1-6 alleles for each marker, and a total of 56 alleles was found: 28 within the NBE sample and 28 within the BE sample. High level of heterozygosity were observed in both populations which could be a result of sexual reproduction. Five microsatellite loci (Pgestssr059, PgtCAA80, Pgestssr293, Pgestssr325, Pgestssr318) were useful to track the origin of Pgt isolates. The NBE sample is characterized by two Pgestssr059 alleles (210 and 228 bp) in a heterozygous state; PgtCAA80 allele of 189 bp; Pgestssr293 allele of $257 \mathrm{bp}$ in a homozygous state; Pgestssr325 allele of $259 \mathrm{bp}$ in a homozygous state. The BE sample is characterized by Pgestssr059 allele of 231 bp in a homozygous state; two Pgestssr293 alleles $(266,269$ bp) in a heterozygous state; two Pgestssr325 (247, 256 bp) in a heterozygous state; Pgestssr318 allele of $207 \mathrm{bp}$.

Acknowledgements: The study was funded by the budgetary project of ICG SB RAS No. 0259-2019-0001, RFBR grant No. 18-016-00170a, the budgetary project of ARRIP No. 0598-2019-0001. 